\title{
Modeling the Dynamics of the Human Thigh for a Realistic Echographic Simulator with Force Feedback
}

\author{
D. d'Aulignac ${ }^{1}$, M. C. Cavusoglu ${ }^{2}$, and C. Laugier ${ }^{1}$ \\ 1 GRAVIR Laboratory \\ INRIA Rhône Alpes \\ 38330 Montbonnot Saint-Martin, France \\ \{Diego.D_Aulignac, Christian.Laugier\}@inrialpes.fr \\ 2 Robotics and Intelligent Machines Laboratory \\ University of California, Berkeley \\ Berkeley, CA 94720 \\ mcenk@eecs. berkeley.edu
}

\begin{abstract}
This paper proposes a mass-spring model of the dynamics of a human thigh based on real data acquired. Using a force sensor mounted on a robot arm the deformation of the thigh with respect to an external force is measured. The stress-strain curves we obtained exhibit a strong non-linearity due to the incompressibility of the human tissue. Hence, we propose a two-layer model of the thigh using both linear and non-linear visco-elastic springs to simulate the observed behaviour. The parameters of the springs are estimated using a least-squares minimisation method. Finally, we discuss the feasibility of our model as part of a fully functional simulator coupled with a haptic interface to train practitioners for echographic exams.
\end{abstract}

\section{Introduction}

Echography, in general terms, exploits the information which an acoustic signal provides when it is reflected off a structure to determine the position and shape of the latter. The frequency of these acoustic signals lie within the range of $1 \mathrm{MHz}$ and $12 \mathrm{MHz}$, thus higher then the sounds which are perceptible by humans. Therefore, they are also referred to as ultrasounds.

Even though they had already been discovered by P. Curie in 1880, they would have to wait until the 1970's to find an application in the field of medicine. Since, they have been widely used as an inexpensive and non-traumatic means of diagnosis. A common exam is the echography of the thigh to detect a thrombosis in the vein. A healthy vein will compress under the influence of an external force while a vein affected by thrombosis will only partially or even not at all compress, depending on the stage in the evolution of the illness. Depending on the pressure the practitioner applies with the echographic probe on the thigh, he will get an image from which the current state of the vein can be deduced, and hence a possible thrombosis diagnosed. 
However, the learning process of this procedure is somehow long and only after approximately 1000 echographic exams an acceptable competence in acquired. The first 500 exams will have to be carried out under the supervision of an experienced practitioner. Virtual environments present an alternative to the conventional medical training scheme. It is possible to create an interactive 3D simulation environment, where the doctors can manipulate or cut dynamically and geometrically correct models of organs and tissues with an haptic interface. The idea is similar to using flight simulators to train pilots. Virtual environments give an environment where there is no risk to a patient, and therefore less stressful. They are interactive and three dimensional contrary to books. Virtual environments also give a unique advantage, as it is possible to generate arbitrary anatomies and pathologies, so that the doctors can be trained for cases that are not frequently encountered.

The goal of this work is to lay the groundwork for the development of an echographic simulator with force feedback. In the final system, the trainee will be looking at artificially generated echographic images, and interacting with a computer simulated dynamical thigh model through a haptic interface. Constructing realistic but computationally efficient models is the main challenge in developing a virtual reality training simulator. In this application it is necessary to have models for deformable tissue being manipulated by the doctor as well as models to construct artificial echographic images.

In this paper, a dynamic model of the human thigh based on experimentally determined deformation characteristics will be presented, followed by a discussion of the results and future directions.

\section{Previous Work}

Henry [4] examined how a set of echographic images could be used to construct a more general model that would take into account the orientation and pressure exerted with the probe on the thigh. The approach is based on an interpolation method; from a real set of echographic images taken, it will build an echographic image for any position and orientation of the probe (see Figure 1 and 2$)^{1}$. Further it is possible to deform the interpolated image obtained with respect to the pressure that is applied to the probe. The criteria on which this deformation depends includes important factors such as the arterial and venous pressure. By modifying these criteria we can simulate a set of pathologies on which medical students could be trained for the identification of the latter.

However, for a meaningful echographic simulation it is of paramount importance to consider the forces involved in such a procedure since they will dictate the deformation of the tissue, and therefore, the subsequent image that is acquired. Laffont [7] was the first to study the implications of the dynamics of such a system which are essential in the development of a realistic simulator coupled to a haptic interface. He based his model on a system of inter-connected springs.

\footnotetext{
${ }^{1}$ Figures kindly provided by the TIMC-GMCAO project
} 


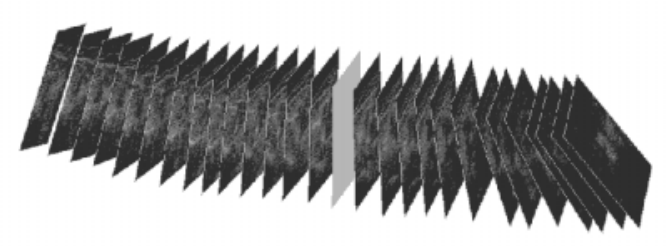

Fig. 1. Creating an image from a set of real echographic images. The highlighted image was obtained by interpolation.
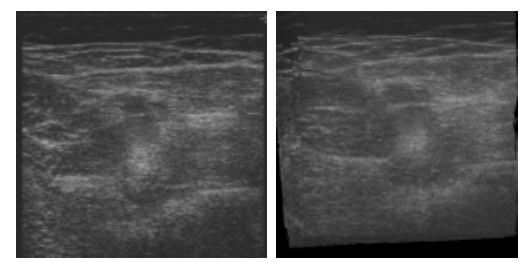

Fig. 2. On the left we can see the real image, and on the right the image obtained by interpolation.

Such a type of approach has already been successfully applied to areas such as facial animation [8] and have the decisive advantage of being easy-to-implement and faster than finite-element simulation, even though lately considerable speedups have been achieved in this domain through the use of pre-calculated matrices. This has resulted in, for example, the real-time simulation with force-feedback of the human liver [2] based on the Visible Human dataset. However, the model used is inconvenient in the sense that the assumption is made that the tissue is hyper-elastic and linear in its deformation. This is clearly not the case. As bio-mechanical experiments confirm the stress-strain curve of human tissue is non-linear. [8] tackles this problem by using a three-layer model which takes into account the properties of the epidermis, the fatty sub-cutaneous tissue, the muscle, and the bone. The great difficulty for such a system lies in the identification of the parameters of the individual springs that will give the same results to an external force being applied as the measurements in the real world.

\section{Modeling of the Thigh}

We have constructed a dynamic model of the human thigh based on experimental measurements of its elasticity.

\subsection{Experimental Setup and Data Acquisition}

In order to model an object such that its behaviour corresponds to reality, measurements must be taken on the real object. In this case we are interested in the 
deformation of the thigh with respect to an external force which is applied. Intuitively we can affirm that the deformation of the thigh is not the same depending on the shape of the object used to provoke this deformation, or more precisely, the contact surface of that object. Since our aim is to build a generic simulation which will allow a physically correct behaviour which is almost independent of the object we choose to deform the thigh with, two different objects have been used to measure the behaviour of the thigh in terms of penetration distance with respect to the external force being applied.
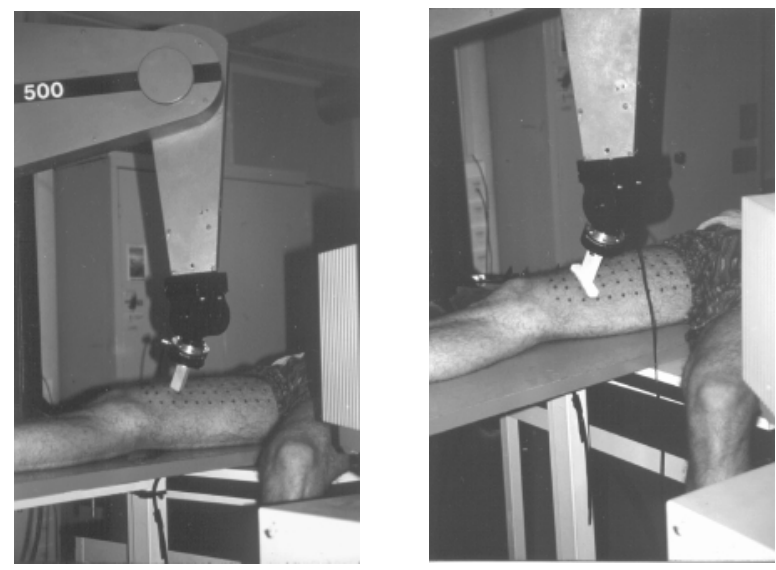

Fig. 3. The two probes used for measuring the behaviour of the thigh.

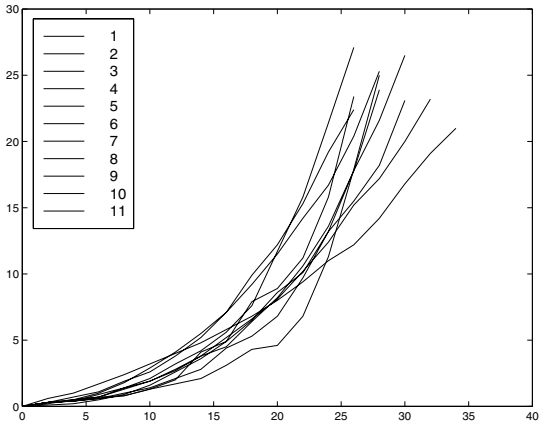

(a)

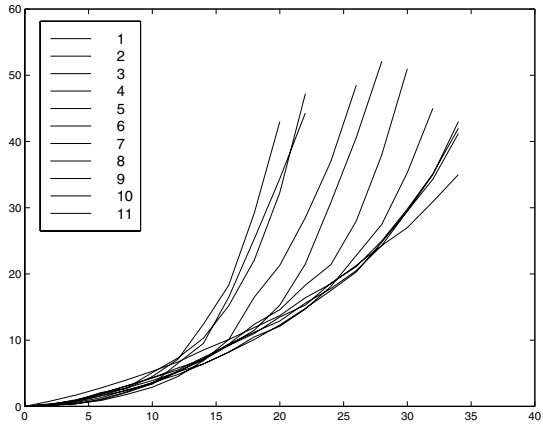

(b)

Fig. 4. Plot of the reaction force in function of the penetration distance at 11 different points on the thigh using a probe with a punctual contact (on the left) and the pseudo-echographic probe with a larger contact surface (on the right).

The first of these has a tip of pyramidal shape to provoke a punctual force response, while the second one has the same contact surface area as a typical echographic probe. These pseudo-probes are then mounted on a force sensor 
which in turn is mounted on a PUMA articulated arm. The probe is then positioned perpendicularly to the surface of the thigh at each of 64 points where measurements will be taken. These points are regularly distributed over the area where the echography is performed when trying to detect a thrombosis in the vein. The robotic arm then advances $2 \mathrm{~mm}$ using the reference of the end effector, i.e. the probe pushes along the axis which is normal to the surface of the thigh at the given point. The force is recorded and the procedure is repeated up to an upper force limit.

Figure 4a shows the non-linear relationship between the penetration distance and the reaction force at 11 different points along the thigh. The difference in the curves can be accounted for due to the fact that the thigh is not homogeneous (e.g. in some regions fatty tissue might be pre-dominant, while in others there may be very little separating the epidermis from the bone).

Figure $4 \mathrm{~b}$ plots the values of the forces for the same points as above but using the second probe with a larger contact surface. As one might expect this results in a larger force for the same penetration distance since the external force applied is distributed over a much larger area.

The assumption is that from these two distinct sets of data it will be possible to make a model which will respond correctly to not only to the two probes used for measurement purposes, but also other probes of a different shape.

\subsection{Model Construction}

Based on the experimental data and the computational requirements, a two layer lumped element model is chosen. The two layer model is composed of a surface mesh of masses, linear springs and dampers, and a set of nonlinear springs orthogonal to the surface to model volumetric effects by giving normal support to the surface mesh (See Figure 5). Two layer models were also used by several authors in the literature, for example in [8]. The deformation-force relation of the nonlinear springs are of the form

$$
f(x)=\frac{x}{a x+b}
$$

The nonlinear spring response is chosen to model the incompressibility of the thigh after a certain deformation. In the model, the values of the surface elements are chosen uniform whereas the parameters of the nonlinear spring vary around the mesh to model heterogeneous nature of the thigh mentioned above, while keeping the number of unknown parameters small.

Parameter Estimation Estimation of the model parameters from experimental measurements is a critical part of the modeling. We have used a two step optimisation approach based on nonlinear least squares estimation. In the two step approach, the experimental data is first fit to a simple model without the surface elements, and the results of this fit is used as the initial conditions for the estimation of the parameters of the complete model. This approach is chosen to avoid problems with local minima. 


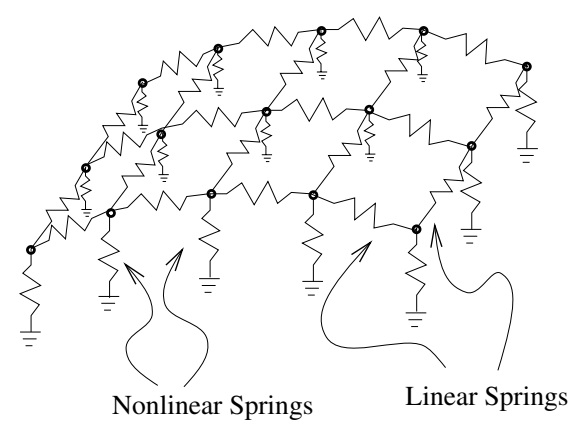

Fig. 5. Two layer model of the thigh.

One thing to note here is that the parameter estimation is based on a simplified interaction model between the tissue and indentor, making the assumption that the probe will principally act on one spring, since the contact area is small. In this simplified interaction model, the nodes that are not in contact with the probe are kept stationary. This simplifies the force calculation as only a small number of springs are deformed for any given dataset.

The mean absolute error between the measured values and the values estimated by the model is 1.05 Newtons, with standard deviation 1.84 Newtons, over the whole dataset. This is equivalent to an approximate error of 5 percent. The distribution of the error can be seen in Figure 6, showing how many of the values estimated by the model exhibit a given error with respect to the real measurements.

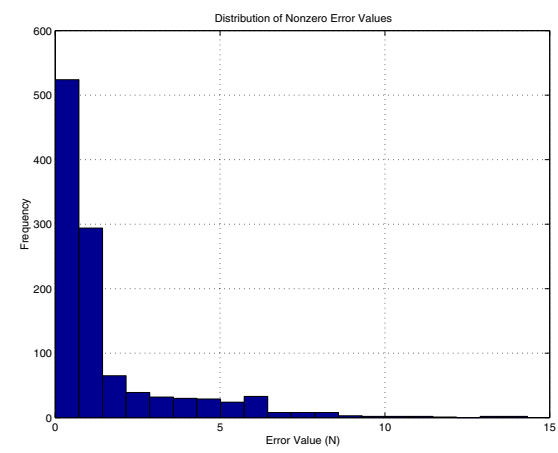

Fig. 6. Frequency distribution of the error between the measured values and the values estimated by the model.

\section{Results}

Figure 7 shows the model as it has been built in our simulation system [5]. A force is being applied on the thigh using a probe which provokes a deformation which is in accordance with the measurements taken. For collision detection 
between the objects the algorithm outlined in [6] is used, while the response to a collision is determined by the approach described in [3].

For the integration of the dynamic, differential equations we use implicit integration as discussed in [1] and using a conjugate gradient solver [9]. This approach compares favourably to the well-known explicit methods in cases where springs are stiff, allowing to take bigger timesteps, and therefore, gain in overall execution time.
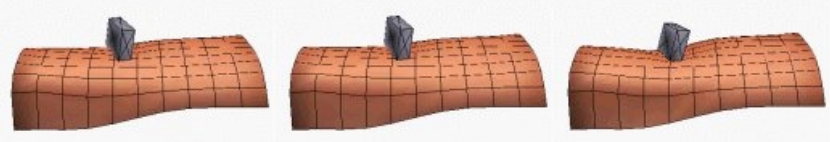

Fig. 7. Model viewed on the simulation system's graphical interface.

The computational speed on a Silicon Graphics R10000 machine is in the order of 100 frames per second of animation, which lets us envisage our simulation system as part of a working echographic simulator which operates in real-time.

\section{Discussion}

We are at the stage where we can simulate accurately the deformation of the thigh under a static load for a variety of objects used to provoke this deformation. However, what is missing are measurements about the damping and friction coefficients. The first is important in the sense that it defines how fast the tissue will react to a change in external force being applied to it, and the latter due to the fact that the practitioner slides the probe down the inner side of the thigh to capture the echographic images. This friction coefficient is influenced by the gel that is typically applied on the thigh to avoid the presence of air between the probe and the tissue, which in turn might influence the quality of the echographic images that are acquired.

We believe that these coefficients may either be adjusted manually using the feedback provided by an experienced practitioner or that additional measurements may be necessary. Once these last problems resolved the primary goal will be to integrate the resulting model with the echographic image creator presented in Section 2 and a force feedback device of type PHANToM built by SensAble Technologies.

\section{Conclusions}

In this paper we have presented and constructed a model of a human thigh from measurements taken in the real world. We emphasise the point that the human thigh is not linear in its elasticity and introduce the use of non-linear springs for this application and propose a possible solution for the identification of the parameters of the springs. Further, from experimental results of the simulation 
using the proposed model we believe in the feasibility of our approach as a part of a real-time system which will allow realistic force feedback and the automatic generation of echographic images which takes into account the current deformation.

\section{Acknowledgements}

We would like to thank Etienne Dombre and François Pierrot at the LIRMM laboratory (Montpellier, France) for their kind assistance while taking the measurements at their lab. Also thanks to the GMCAO project at the TIMC lab in Grenoble, and in particular to Jocelyne Troccaz for her help. This work was partially supported by an INRIA grant within the framework of the AISIM initiative and the France-Berkeley Fund.

Related Web Sites For more information on the project visit our project homepage: http://www.inrialpes.fr/sharp/modelisation/index-en.html

\section{References}

1. D. Baraff and A. Witkin. Large steps in cloth simulation. In Computer Graphics (Proc. SIGGRAPH), pages 43-54, 1998. 1197

2. M. Bro-Nielsen and S. Cotin. Real-time volumetric deformable models for surgery simulation using finite elements and condensation. In Proceedings of Eurographics, volume 15, pages 57-66, 1996. 1193

3. A. Deguet, A. Joukhadar, and C. Laugier. Models and algorithms for the collision of rigid and deformable bodies. In P. K. Agarwal, L. E. Kavraki, and M. T. Mason, editors, Robotics: the algorithmic perspective, pages 327-338. A K Peters, 1998. Proc. of the Workshop on the Algorithmic Foundations of Robotics. Houston, TX (US). March 1998. 1197

4. D. Henry. Outils pour la modélisation de structure et la simulation d'examens échographiques. PhD thesis, Université Joseph Fourier, Grenoble (FR), 1997. (in french). 1192

5. A. Joukhadar and C. Laugier. Dynamic simulation: Model, basic algorithms, and optimization. In Proc. of the Workshop on the Algorithmic Foundations of Robotics, Toulouse (FR), July 1996. 1196

6. A. Joukhadar, A. Wabbi, and C. Laugier. Fast contact localisation between deformable polyhedra in motion. In Proc. of the IEEE Computer Animation Conf., pages 126-135, Geneva (CH), June 1996. 1197

7. P. Laffont. Simulation dynamique pour le diagnostic de thromboses veineuses. Mémoire de Diplôme d'Etudes Approfondies, Université de Savoie, Chambéry (FR), 1997. 1192

8. Y. Lee, D. Terzopoulos, and K. Waters. Realistic facial modeling for animation. In Computer Graphics Proceedings, Annual Conference Series, Proc. SIGGRAPH '95 (Los Angeles, CA), pages 55-62. ACM SIGGRAPH, August 1995. 1193, 1195

9. W. H. Press, B. P. Flannery, S. A. Teukolsky, and W. T. Vetterling. Numerical Recipes in C. Cambridge Univ. Press, 2 edition, 1992. 1197 